\title{
Nikitas Aliprantis, Les héritages cruciaux du XXe siècle aux sociétés d'aujourd'hui
}

Jean-Michel Belorgey

\section{OpenEdition}

1 Journals

\section{Édition électronique}

URL : https://journals.openedition.org/rdctss/2066

DOI : 10.4000/rdctss.2066

ISSN : 2262-9815

Éditeur

Centre de droit comparé du travail et de la sécurité sociale

\section{Édition imprimée}

Date de publication : 1 avril 2018

Pagination : 198-202

ISSN : 2117-4350

\section{Référence électronique}

Jean-Michel Belorgey, «Nikitas Aliprantis, Les héritages cruciaux du XXe siècle aux sociétés

d'aujourd'hui », Revue de droit comparé du travail et de la sécurité sociale [En ligne], 1 | 2018, mis en ligne le 01 novembre 2021, consulté le 12 novembre 2021. URL : http://journals.openedition.org/rdctss/ 2066 ; DOI : https://doi.org/10.4000/rdctss.2066

\section{cc) (†) $९$}

Revue de droit comparé du travail et de la sécurité sociale est mise à disposition selon les termes de la Licence Creative Commons Attribution - Pas d'Utilisation Commerciale - Pas de Modification 4.0 International. 


\section{ChroniQue BIBLIOGRAPHIQUE}
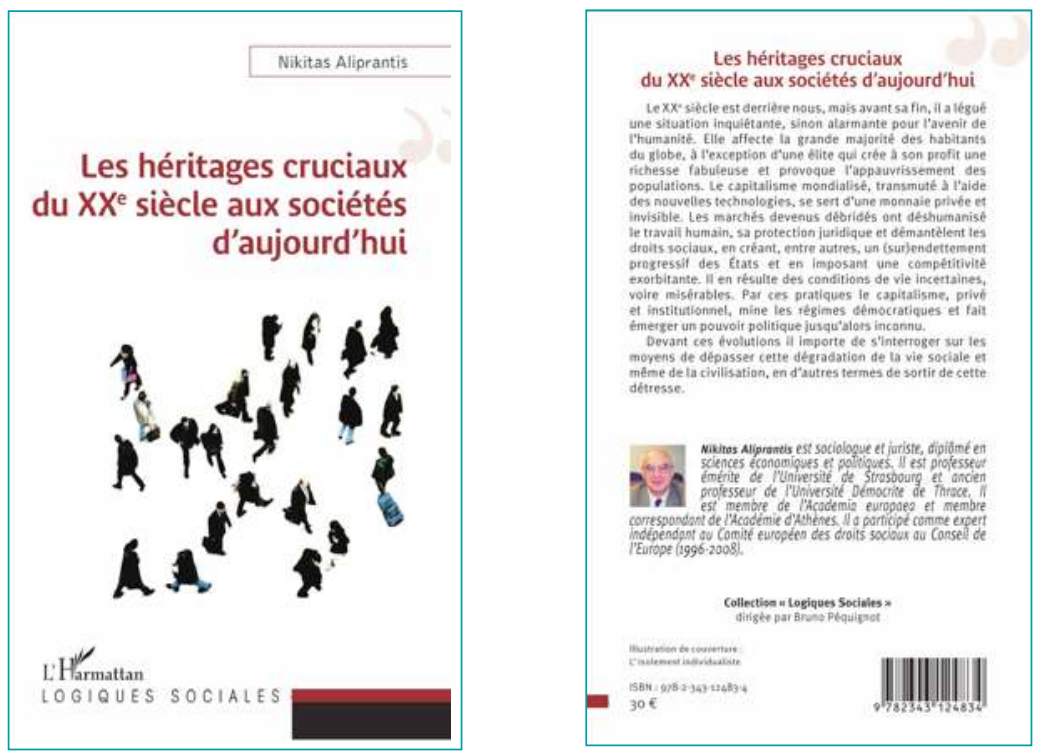

\section{NiKitas Aliprantis \\ LES HÉRITAGES CRUCIAUX DU XX' SIÈCLE AUX SOCIÉTÉS D'AUJOURD'HUI \\ Par Jean-Michel Belorgey \\ Président de section honoraire au Conseil d'État}

C'était, pour l'avoir côtoyé pendant quelques dix ans au sein du Comité européen des droits sociaux du Conseil de l'Europe (organe de régulation de la Charte sociale européenne, qu'il ne faut pas confondre avec la Charte des droits fondamentaux de l'Union, laquelle ne reprend qu'une faible partie des prévisions de la précédente, sur un mode souvent ambigu) dont nous étions membres tous les deux, essentiellement comme juriste que, avant de lire l'ouvrage qu'il vient de publier en français, l'une des langues que ce polyglotte pratique couramment, j'étais enclin à percevoir le professeur Nikitas Aliprantis. Bien que j'ai lu, quand il m'en a fait hommage, au début des années 2000, son ouvrage sur Les organisations patronales et la justice en matière sociale ${ }^{1}$ dont l'élogieuse préface par le professeur Gérard Lyon-Caen, je la redécouvre, débute en ces termes: «Voici une étude que nos juristes auraient tort d'ignorer encore qu'elle se situe plutôt du côté de la sociologie judiciaire que de la sociologie du travail ». Bien que les Mélanges réalisés en 2014 en 
son honneur ${ }^{2}$ regroupent essentiellement, dans leur seconde partie, des contributions de sociologues. Il ne m'en a toujours pas moins paru évident que Nikitas Aliprantis ne partageait pas l'allergie maladive de nombre de juristes, et même de philosophes du droit, à l'égard des sciences sociales, allergie dont il m'a bien fallu m'accommoder quand, rapporteur général de la section du rapport et des études du Conseil d'État, et soumis à la relecture pour validation de mes projets de rapport, l'un de mes collègues d'âge respectable protestait «ce n'est pas du droit, c'est de la sociologie », ce qui n'était, hélas, pas le propre d'une génération, puisqu'un jeune rapporteur à qui je suggérais la lecture d'un livre de sciences sociales sur les questions de précarité et de pauvreté me rétorquait, presque indigné, "moi je fais du droit, monsieur ». Je n'ai plus de doute désormais que, distingué juriste, Nikitas Aliprentis soit aussi un distingué sociologue; et il se pourrait que ceci ne soit pas un hasard, que cette double compétence soit la clef d'une compréhension point trop mutilée de nombre de faits sociaux, et d'une capacité à avoir sur eux une véritable prise (l'imaginaire du droit, des juristes et des non juristes, y compris des philosophes des sciences visités par une fascination ambigüe du droit - Bruno Latour et « La fabrique du droit » - a récemment donné lieu à de belles réflexions ${ }^{3}$ ).

Compréhension: il faut prendre garde aux mots quand on traite de sociologie comme quand on fait du droit; Nikitas Aliprantis, dans la première partie de son ouvrage, en même temps qu'il fait rappel des différentes étapes de l'évolution de la réflexion sociologique au cours du dernier siècle, des relations entre philosophie et sociologie, des débats entre écoles, empiristes et hyper empiristes, modernistes et post modernistes, évoque d'ailleurs la proposition webérienne de dépasser l'opposition de Dilthey entre compréhension, qui serait l'affaire des sciences sociales, et explication, qui serait l'affaire des sciences de la nature, par la notion d'explication compréhensive. C'est bien, semble-t-il, sous le signe de ce dépassement ainsi que d'une fidélité à l'approche critique d'Habermas, tant dans l'attention portée à la langue que dans l'appel à des concepts économiques d'origine marxiste que se situe la suite de l'ouvrage.

Le fait est que "Les héritages cruciaux du XX'̀me siècle aux sociétés d'aujourd'hui » (on aurait préféré un titre plus porteur) n'est pas qu'un inventaire des multiples dérives tant des politiques publiques que des représentations gouvernant largement celles-ci, avec leur cortège de conséquences en termes d'effritement du lien social, ceci non seulement dans les domaines dont l'auteur est familier, travail, protection sociale, santé, mais dans d'autres, aussi divers que l'environnement, les migrations, la sécurité, les relations avec le Tiers monde. Il s'agit aussi, d'évidence, d'un livre de passion. Plût au ciel que personne ne s'avise d'en faire reproche à Nikitas Aliprantis, comme certains de ses élèves, au cours d'une séance dont j'ai gardée un souvenir amer, l'ont fait à Georges Friedmann, après la publication, en 1970, de La puissance et la sagesse.

2 Le travail humain, au carrefour du droit et de la sociologie, hommage au professeur Nikitas Aliprantis, Presses universitaires de Strasbourg, 2014

3 Voir M. Doat et G. Darcy (dir.), L'imaginaire en droit, coll. Penser le droit, qui se définit comme accueillant des ouvrages originaux de philosophie et de théorie du droit, éd. Bruylant. Bruxelles, 2011. Et Liber Amicorum Darcy, Détours juridiques: le praticien, le théoricien et le rêveur, coordonné par Mathieu Doat et Patrick Charlot, éditions Bruylant, Bruxelles 2012, qui contient un splendide bouquet de contributions juridiques, philosophiques et sociologiques. 
Des analyses proposées, il ressort très clairement que le legs aux sociétés contemporaines du XXème siècle finissant procède pour une large part du reniement de nombre d'avancées de la pensée et de la pratique qui avaient pu se faire jour au lendemain de la seconde guerre mondiale sous l'effet d'une réaction contre les formes de sauvagerie et de perversion mentale dont elle avait permis l'expression, au bénéfice peut-être d'une période de prospérité peu ou prou partagée, mais aussi grâce à l'épanouissement d'ambitions humanistes susceptibles d'être portées par des forces sociales d'horizons philosophiques très divers. Le mouvement engagé au lendemain de l'écrasement de l'Allemagne hitlérienne n'a malheureusement pas été durable et s'est peu à peu essoufflé. Non seulement, l'empire soviétique, mais encore l'empire américain, et même les démocraties occidentales se sont peu à peu lassées de conduire des politiques à la mesure des valeurs brandies. La capacité des États, qui avaient peut-être voulu trop embrasser, à jouer, plus modestement, mais honorablement, le rôle d'arbitre, en contenant, à défaut de faire reculer, les inégalités, s'est elle-même trouvée entamée, du fait de la montée d'autres acteurs sur les scènes nationales et sur la scène internationale, de l'influence croissante de ceux-ci sur la décision publique, de la pénétration jusque dans les appareils d'État des idéologies les animant. La construction européenne n'a pas échappé à ces influences, ni à l'infléchissement des politiques qu'elles entendaient et sont parvenues à promouvoir au nom de formes de rationalités présentées comme modernes alors qu'elles n'étaient souvent qu'archaïques, et, en fin de compte, d'une abjuration parfois déguisée, parfois, et de plus en plus, cynique, des valeurs regardées quelques décennies plus tôt comme propres à fonder le bien-être collectif et la cohésion sociale.

C'est de tout cela dont il est question au fil des pages de l'ouvrage de Nikitas Aliprantis qui, bien qu'à dominante sociologique, ne tourne pas le dos au droit, ne serait-ce que pour identifier les conséquences sur l'évolution des rapports au droit, et sur celle du droit, des lames de fond d'ordre psychologique, économique et sociologique (retour à un culte effréné du profit, financiarisation de l'économie, valorisation inconditionnelle de l'idée de compétition et dévalorisation de celle de solidarité, tolérance ou encouragement d'un nouveau dualisme social), qui ont bouleversé le paysage mental et social dans le dernier tiers du XXème siècle. Des formes de méconnaissance ou de mépris du droit et des droits, y compris des droits internationalement reconnus, mais peu à peu bafoués, de front ou au prix de différentes catégories de contournements, par des législations progressivement soumises à des révisions drastiques (ou cédant à des tentations abstentionnistes ou parodiques: le droit mou), l'ouvrage fournit plusieurs exemples ${ }^{4}$ Y compris celui, plus attristant que d'autres, du droit de l'Union européenne, et, hors de tout droit, celui de ses coups de force, notamment contre la Grèce ${ }^{5}$.

Sur le front du travail, qui, malgré la diversification de ses formes, telle que l'ont mise en évidence plusieurs auteurs, notamment Alain Supiot, et de la difficulté qui en résulte à lui conférer un statut approprié, reste confusément, ou obsessionnellement, sous ses formes classiques (le salariat) ou dégradées (sous-traitances, travail à domicile) la référence de la plupart des décideurs (Tony Blair faisant valoir au Hilton de Londres, lors d'un séminaire

4 L'un des plus horribles est celui de la confiscation par le Danemark des objets précieux des immigrants, y compris les demandeurs d'asile. Le Danemark n'a pas résilié, que l'on sache, son adhésion à la Convention de Genève, ni à la Convention européenne des droits de l'homme.

5 Plusieurs contributions, dont la mienne, traitent de cette question dans les Mélanges précités en I'honneur de Nikitas Aliprantis. 
sur la pauvreté, que l'homme doit gagner son pain à la sueur de son front, et qu'il n'y a pas de limite inférieure à la rémunération du travail), alors que ceux-ci se révèlent impuissants à fixer des bornes tant à la flexibilité des durées et des horaires de travail qu'à sa surintensification, complices aussi de l'individualisation croissante des relations de travail, laissant les travailleurs les moins bien armés démunis face à leurs interlocuteurs patronaux.

Sur le front de l'environnement, où, malgré les réticences d'un certain nombre de parties prenantes, la formulation d'une nouvelle génération de droits s'était progressivement esquissée, au nom de l'idée qu'il fallait mettre un frein à la production de biens au prix de la dégradation de la santé des travailleurs et des riverains, de la destruction des milieux naturels; qu'il fallait que les bénéfices d'une telle production cessent d'être maximisés du fait d'une externalisation aux dépens de la collectivité, et des générations futures, de coûts incombant aux producteurs. Les générations futures, et la belle idée de droit des générations futures! Le malheur est que l'économie du profit, sa financiarisation, l'ubérisation d'une partie des relations sociales, ne privilégient pas le long mais le court terme. «Après moi (et autour de moi), le déluge » : ce sont là la vision du monde et la politique de Trump, mais pas lui seulement, de toute une génération de dirigeants; d'où le rejet par le premier des accords internationaux sur le climat, sur le commerce de l'ivoire, et le refus de réglementer la vente d'armes sur le territoire américain malgré les hécatombes n'ayant pas pour origine un terrorisme plus ou moins politique mais le recours à la violence comme seul exutoire, avec, souligne Nikitas Aliprantis, l'approbation - pour la tuerie norvégienne - de quelques auteurs hallucinés.

Sur le front du néo-colonialisme et des rapports avec le Tiers monde, où les indépendances n'ont pas mis fin aux pillages, ni aux ingérences (rarement aussi vertueuses que le suggère l'idée de devoir d'ingérence) mais multiplié les clientélismes entre les anciens impérialistes et tous les échelons des gouvernements locaux, sans oublier les alliances contre nature et les inventions d'apprentis-sorciers (Al Qaïda).

Sur le front de la sécurité (comprise au sens étroit de protection des biens et des personnes contre les atteintes terroristes ou de bandes criminelles, et non contre celles d'autres catégories de délinquants: les États que Libération a récemment qualifiés d'États toxiques, le capitalisme comme source d'insécurité générale). À cet égard, Nikitas Aliprantis insiste tout particulièrement sur les risques que fait courir aux libertés le développement d'un fichage en principe encadré, notamment en France, par une législation appropriée (la législation Informatique et libertés), mais ayant tendance à proliférer non seulement entre les mains de l'État, mais encore des collectivités locales et de différentes catégories d'opérateurs dans des conditions mal maîtrisées.

Dans ses constats conclusifs anthropologiques, l'auteur insiste, une fois de plus utilement, sur le paradoxe que constitue la coexistence d'une victoire apparente de l'individualisme et d'un écrasement de l'individu non seulement au travail, mais dans d'autres dimensions de sa vie, l'accès à la culture, la relation au passé historique, ou tout simplement le dialogue avec ses semblables qui ne soit pas court-circuité par la machine (impossibilité d'un face à face visuel ou même vocal, auxquels font systématiquement obstacle les plateformes téléphoniques des administrations, de la sécurité sociale, de toutes sortes de fournisseurs). J'ai été surpris que, traitant pertinemment des problèmes d'immigration, et des problèmes d'insertion des migrants dans la société d'accueil (intégration des migrants ou multiculturalisme; pourquoi « ou», le multiculturalisme, les multi-appartenances, familles transfrontières et double nationaux, si fort que l'idée révulse nombre de souverainistes dans 
les milieux de pouvoir et ailleurs, n'ouvrent-ils pas de nouvelles perspectives pour le monde à venir ?) il n'évoque pas davantage la place des replis identitaires de toutes espèces parmi les réactions de protection contre le vide existentiel qu'il mentionne à plusieurs reprises, et, en même temps que les dangers, les quelques vertus, tout de même, de ce phénomène; plus encore que, au titre des problèmes d'identité, il ne fasse quasiment pas mention des remous qui, la peur de l'islam aidant, engendrent, en France, le retour à une conception erronée de la laïcité, et plus généralement, ailleurs, des perversions contradictoires de la conception du champ des libertés religieuses.

Bon, pour rarement porteuses d'euphorie que soient les analyses présentées par Nikitas Aliprantis, il a manifestement souhaité ne pas laisser ses lecteurs sans armes contre le désespoir, et un nombre raisonnable de pages prenant place avant la conclusion est consacré, sous l'invocation de "la lutte pour le droit» de R. von Jhering, aux initiatives alternatives positives repérables, notamment l'économie sociale et solidaire dont il est aussi question dans un récent beau livre de Jacques Fournier, "L'économie des besoins", et aux réformes économiques souhaitables (refréner les marchés, lutter contre les paradis fiscaux, imposer les revenus financiers) dont l'auteur ne cache pas que plus d'une soit problématique ... pour les raisons qu'il a précédemment mises en évidence.

Une lecture incontestablement stimulante, faisant consonner approches sociologique, juridique, économique aussi, et, ça n'est un crime que pour les hyper positivistes, philosophique et éthique. Dont il est dommage seulement que l'accès risque d'être rendu indûment difficile par la politique de diffusion de l'éditeur, s'il en a une. 